\title{
Partially Null Curves of Constant Breadth in Semi-Riemannian Space
}

\author{
Süha Y1lmaz \\ Buca Educational Faculty, Dokuz Eylül University \\ 35160, Buca-Izmir, Turkey \\ E-mail: suha.yilmaz@yahoo.com \\ Melih Turgut (Corresponding author) \\ Buca Educational Faculty, Dokuz Eylül University \\ 35160, Buca-Izmir, Turkey \\ E-mail: melih.turgut@gmail.com
}

\begin{abstract}
In this work, some characterizations of partially null curves of constant breadth in Semi-Riemannian space are presented.

Keywords: Semi-Riemannian Space, Partially Null Curves, Curves of Constant Breadth

\section{Introduction}

Curves of constant breadth were introduced by L. Euler, 1780. Ö. Köse (1984) wrote some geometric properties of plane curves of constant breadth. And, in another work Ö. Köse (1986) extended these properties to the Euclidean 3-Space $E^{3}$. Moreover, M. Fujivara (1914) obtained a problem to determine whether there exist space curve of constant breadth or not, and he defined 'breadth' for space curves and obtained these curves on a surface of constant breadth. A. Magden and Ö. Köse (1997) studied this kind curves in four dimensional Euclidean space $E^{4}$.

E. Cartan opened door of notion of null curves (for more details see C.Boyer, 1968). And, thereafter null curves were deeply studied by W.B. Bonnor (1969) in Minkowski space-time. In the same space, special null; Partially and Pseudo Null curves are defined by J. Walrave (1995). Additionally, M. Petrovic et. al. (2005) defined Frenet equations of pseudo null and partially null curves in $E_{2}^{4}$.

In this paper, we extend the notion of curves of constant breadth to null curves in Semi-Riemannian space. Some characterizations are obtained by means of Frenet equations defined by M. Petrovic et. al.(2005). We used the method of Ö. Köse (1984).
\end{abstract}

\section{Preliminary Notes}

To meet the requirements in the next sections, here, the basic elements of the theory of curves in the space $E_{2}^{4}$ are briefly presented. A more complete elementary treatment can be found in B. O’Neill (1983).

Semi-Riemannian space $E_{2}^{4}$ is an Euclidean space $E^{4}$ provided with the standard flat metric given by

$g=-d x_{1}^{2}-d x_{2}^{2}+d x_{3}^{2}+d x_{4}^{2}$,

where $\left(x_{1}, x_{2}, x_{3}, x_{4}\right)$ is a rectangular coordinate system in $E_{2}^{4}$.

Since $g$ is an indefinite metric, recall that a vector $\vec{v} \in E_{2}^{4}$ can have one of the three causal characters; it can be space-like if $g(\vec{v}, \vec{v})\rangle 0$ or $\vec{v}=0$, time-like if $g(\vec{v}, \vec{v})\langle 0$ and null (light-like) if $g(\vec{v}, \vec{v})=0$ and $\vec{v} \neq 0$. Similarly, an arbitrary curve $\vec{\alpha}=\vec{\alpha}(s)$ in $E_{2}^{4}$ can be locally be space-like, time-like or null (light-like), if all of its velocity vectors $\vec{\alpha}^{\prime}(s)$ are respectively space-like, time-like or null. Also, recall the norm of a vector $\vec{v}$ is given by $\|\vec{v}\|=\sqrt{|g(\vec{v}, \vec{v})|}$. Therefore, $\vec{v}$ is a unit vector if $g(\vec{v}, \vec{v})= \pm 1$. Next, vectors $\vec{v}, \vec{w}$ in $E_{2}^{4}$ are said to be orthogonal if $g(v, w)=0$. The velocity of the curve $\vec{\alpha}$ is given by $\left\|\vec{\alpha}^{\prime}\right\|$. Thus, a space-like or a time-like curve $\vec{\alpha}$ is said to be parametrized by arclength function $s$, if $g\left(\vec{\alpha}^{\prime}, \vec{\alpha}^{\prime}\right)= \pm 1$. Let $\vec{a}, \vec{b}$ be two space-like vectors in $E_{2}^{4}$, then, there is unique real 
number $0 \leq \delta \leq \pi$, called angle between $\vec{a}$ and $\vec{b}$, such that $g(\vec{a}, \vec{b})=\|\vec{a}\|\|\vec{b}\| \cos \delta$.

Denote by $\left\{\vec{T}(s), \vec{N}(s), \vec{B}_{1}(s), \vec{B}_{2}(s)\right\}$ the moving Frenet frame along the curve $\vec{\alpha}$ in the space $E_{2}^{4}$. Then $\vec{T}, \vec{N}, B_{1}, B_{2}$ are, respectively, the tangent, the principal normal, the first binormal and the second binormal vector fields. Recall that a space-like curve with time-like principal normal $\vec{N}$ and null first and second binormal is called a partially null curve in $E_{2}^{4}$ M. Petrovic et. al. (2005). Let $\vec{\alpha}$ be a partially null curve in the space $E_{2}^{4}$, parametrized by arclength function $s$. Then for the curve $\vec{\alpha}$ the following Frenet equations are given by M. Petrovic et. al. (2005):

$$
\left[\begin{array}{c}
\vec{T}^{\prime} \\
\vec{N}^{\prime} \\
\vec{B}_{1}^{\prime} \\
\vec{B}_{2}^{\prime}
\end{array}\right]=\left[\begin{array}{cccc}
0 & \kappa & 0 & 0 \\
\kappa & 0 & \tau & 0 \\
0 & 0 & \sigma & 0 \\
0 & \tau & 0 & -\sigma
\end{array}\right]\left[\begin{array}{l}
\vec{T} \\
\vec{N} \\
\vec{B}_{1} \\
\vec{B}_{2}
\end{array}\right]
$$

where $\vec{T}, \vec{N}, B_{1}$ and $\vec{B}_{2}$ are mutually orthogonal vectors satisfying equation $g(\vec{T}, \vec{T})=1, g(\vec{N}, \vec{N})=-1, g\left(\vec{B}_{1}, \vec{B}_{1}\right)=g\left(\vec{B}_{2}, \vec{B}_{2}\right)=0, g\left(\vec{B}_{1}, \vec{B}_{2}\right)=1$,

And here, $\kappa(s), \tau(s)$ and $\sigma(s)$ are first, second and third curvature of the curve $\vec{\alpha}$, respectively.

M. Petrovic et. al. (2005) gave a characterization about partially null curves with the following statement.

Theorem 2.1 A partially null unit speed curve $\vec{\varphi}=\vec{\varphi}(s)$ in $E_{2}^{4}$, with curvatures $\kappa \neq 0, \tau \neq 0$ for each $s \in I \subset R$ has $\sigma=0$ for each $s$.

\section{Partially Null Curves of Constant Breadth in $E_{2}^{4}$}

Let $\vec{\varphi}=\vec{\varphi}(s)$ be a simple closed partially null curve in the space $E_{2}^{4}$. These curves will be denoted by $C$. The normal plane at every point $P$ on the curve meets the curve at a single point $Q$ other than $P$. We call the point $Q$ the opposite point of $P$. We consider a partially null curve in the class $\Gamma$ as in M. Fujivara (1914) having parallel tangents $\vec{T}$ and $\vec{T}^{*}$ in opposite directions at the opposite points $\vec{\varphi}$ and $\vec{\varphi}^{*}$ of the curve. A simple closed curve of constant breadth having parallel tangents in opposite directions at opposite points can be represented with respect to Frenet frame by the equation

$$
\vec{\varphi}^{*}=\vec{\varphi}+m_{1} \vec{T}+m_{2} \vec{N}+m_{3} \vec{B}_{1}+m_{4} \vec{B}_{2},
$$

where $m_{i}(s), 1 \leq i \leq 4$ arbitrary functions of $s$ and $\vec{\varphi}$ and $\vec{\varphi}^{*}$ are opposite points. Vector $\vec{d}=\vec{\varphi}^{*}-\vec{\varphi} \quad$ is called 'the distance vector' of $C$. Differentiating both sides of (1) and considering Frenet equations, we have

$$
\frac{d \vec{\varphi}^{*}}{d s}=\vec{T}^{*} \frac{d s^{*}}{d s}=\left(\frac{d m_{1}}{d s}+m_{2} \kappa+1\right) \vec{T}+\left(\frac{d m_{2}}{d s}+m_{1} \kappa+m_{4} \tau\right) \vec{N}+\left(\frac{d m_{3}}{d s}+m_{2} \tau\right) \vec{B}_{1}+\left(\frac{d m_{4}}{d s}\right) \vec{B}_{2} .
$$

Since $\vec{T}^{*}=-\vec{T}$. Rewriting (2), we obtain following system of equations,

$$
\left(\begin{array}{l}
\frac{d m_{1}}{d s}+m_{2} \kappa+1+\frac{d s^{*}}{d s}=0 \\
\frac{d m_{2}}{d s}+m_{1} \kappa+m_{4} \tau=0 \\
\frac{d m_{3}}{d s}+m_{2} \tau=0 \\
\frac{d m_{4}}{d s}=0
\end{array}\right)
$$

If we call $\phi$ as the angle between the tangent of the curve $C$ at point $\vec{\varphi}$ with a given fixed direction and consider $\frac{d \phi}{d s}=\kappa$, we have (3) as follow: 


$$
\left(\begin{array}{l}
\frac{d m_{1}}{d \phi}=-m_{2}-f(\phi) \\
\frac{d m_{2}}{d \phi}=-m_{1}-m_{4} \rho \tau \\
\frac{d m_{3}}{d \phi}=-m_{2} \rho \tau \\
\frac{d m_{4}}{d \phi}=0
\end{array}\right)
$$

where $f(\phi)=\rho+\rho^{*}, \rho=\frac{1}{\kappa}$ and $\rho^{*}=\frac{1}{\kappa^{*}}$ denote the radius of curvatures at $\vec{\varphi}$ and $\vec{\varphi}^{*}$, respectively. It is not difficult to see that $m_{4}=c_{4}=$ constant. Then, using system (4), we easily have the following differential equations with respect to $m_{1}$ and $m_{2}$ as

$$
\left(\begin{array}{l}
\frac{d^{2} m_{1}}{d \phi^{2}}-m_{1}+\frac{d f}{d \phi}-c_{4} \rho \tau=0 \\
\frac{d^{2} m_{2}}{d \phi^{2}}+m_{2}+c_{4} \frac{d}{d \phi}(\rho \tau)-f(\phi)=0
\end{array}\right)
$$

These equations are characterizations for the curve $\vec{\varphi}^{*}$. If the distance between opposite points of $C$ and $C^{*}$ is constant, then, due to null frame vectors, we can write that

$$
\left\|\vec{\varphi}^{*}-\vec{\varphi}\right\|=m_{1}^{2}-m_{2}^{2}+2 m_{3} m_{4}=l^{2}=\text { constant. }
$$

Hence, by the differentiation we have

$$
m_{1} \frac{d m_{1}}{d \phi}-m_{2} \frac{d m_{2}}{d \phi}+m_{3} \frac{d m_{4}}{d \phi}+m_{4} \frac{d m_{3}}{d \phi}=0
$$

Considering system (4), we get

$$
m_{1}\left(\frac{d m_{1}}{d \phi}+m_{2}\right)=0
$$

Since, we arrive $m_{1}=0$ or $\frac{d m_{1}}{d \phi}=-m_{2}$. Therefore, we shall study in the following cases.

Case 1: $m_{1}=0$. Moreover, let us suppose that $c_{4} \neq 0$. In this case $(5)_{1}$ deduce other components, respectively,

$$
m_{2}=-f(\phi)=-c_{4} \int_{0}^{\phi} \rho \tau d \phi
$$

and

$$
m_{3}=-\int_{0}^{\phi}\left(\rho+\rho^{*}\right) \rho \tau d \phi .
$$

Additionally, it is safe to report that the following relation hold:

$$
\rho^{*}=-\rho-c_{4} \int_{0}^{\phi} \rho \tau d \phi .
$$

And if $c_{4}=0$, we have $f(\phi)=c=$ constant. By this way, we know

$$
\left(\begin{array}{l}
m_{2}=-c \\
m_{3}=c \int_{0}^{\phi} \rho \tau d \phi \\
\rho^{*}+\rho+c=0
\end{array}\right) .
$$


Case 2: $\frac{d m_{1}}{d \phi}=-m_{2}$. In this case, from (4) we know $f(\phi)=m_{2}=0$. And first let us suppose that $c_{4} \neq 0$. Thus the equation $(5)_{1}$ has the form

$$
\frac{d^{2} m_{1}}{d \phi^{2}}-m_{1}=c_{4} \rho \tau .
$$

By the method of variation of parameters, the solution of (13) yields that

$$
m_{1}=e^{\phi}\left[A+\frac{c_{4}}{2} \int_{0}^{\phi} \rho \tau e^{-\phi} d \phi\right]+e^{-\phi}\left[B-\frac{c_{4}}{2} \int_{0}^{\phi} \rho \tau e^{\phi} d \phi\right],
$$

where $A, B$ are real numbers. Denoting $\frac{d m_{1}}{d \phi}=\xi(\phi)$, we have other component

$$
m_{3}=\int_{0}^{\phi} \rho \tau \xi d \phi
$$

Last, we know that

$$
\rho^{*}+\rho=0
$$

And if $c_{4}=0$, we write that

$$
\frac{d^{2} m_{1}}{d \phi^{2}}-m_{1}=0 .
$$

Using hyperbolic functions, we write the solution of (17) as

$$
m_{1}=l_{1} \cosh \phi+l_{2} \sinh \phi .
$$

Considering (4), we have other components,

$$
m_{2}=-l_{1} \sinh \phi-l_{2} \cosh \phi
$$

and

$$
m_{3}=\int_{0}^{\phi}\left(l_{1} \cosh \phi+l_{2} \sinh \phi\right) \rho \tau d \phi .
$$

Case 3: $m_{1}=c_{1}=$ constant $\neq 0$ and $m_{2}=0$. Using (5) 1 , we arrive

$$
\rho \tau=\text { constant. }
$$

Corollary 3.1 In case 3, $c_{4}$ cannot be zero.

\section{References}

A. Magden and Ö. Köse, On The Curves of Constant Breadth, Tr. J. of Mathematics, 1 (1997), 277-284.

B. O'Neill, Semi-Riemannian Geometry, Academic Press, New York, 1983.

C.Boyer, A History of Mathematics, New York: Wiley, 1968.

J. Walrave, Curves and surfaces in Minkowski space. Dissertation, K. U. Leuven, Fac. of Science, Leuven (1995)

L. Euler, De Curvis Trangularibis, Acta Acad. Petropol (1780), 3-30.

M. Fujivara, On Space Curves of Constant Breadth, Tohoku Math. J. 5 (1914), 179-184.

M. Petrovic-Torgasev, K.Ilarslan and E. Nesovic, On partially null and pseudo null curves in the semi-euclidean space $R_{2}^{4}$. J.of Geometry. 84 (2005) 106-116.

Ö. Köse, Some Properties of Ovals and Curves of Constant Width in a Plane, Doga Sci. J. Serial B (8) 2 (1984), 119-126.

Ö. Köse, On Space Curves of Constant Breadth, Doga Turk Math. J. (10) 1 (1986), 11-14.

W. B. Bonnor, Null curves in a Minkowski space-time, Tensor. 20 (1969) 229-242. 